\title{
Nature and Properties of the Chandler Motion and Mechanism of its Damping and Excitation
}

\author{
J. M. Ferrándiz \\ Dept. Matemática Aplicada, University of Alicante, Spain \\ Yu. V. Barkin \\ Sternberg Astronomical Institute, Moscow, Russia
}

\begin{abstract}
The analytical studies of the Chandler motion of the Earth's pole on the basis of the special approach to the problem, using the canonical and noncanonical equations in the Andoyer elastic variables (Barkin, et al. 1995; Barkin, 1996; in press) have been fulfilled. The Earth is considered as an isolated celestial body with the anelastic (in general case) external envelope (the mantle) and an invariant central part (the core).
\end{abstract}

The interpretation of the Chandler motion of the body, deformed by its own rotation, was given in the case of an elastic envelope. It was shown that the body rotates as a fictitious rigid body with different moments of inertia. The analytical solution of the problem let us explain the next properties of the motion of the deformable bodies: 1) observed period of the Earth's polar motion; 2) ellipticity of the pole trajectory and difference of the eccentricities of the Chandler and Euler motions; 3) nonuniform velocity of the counter-clockwise polar motion along the Chandler ellipse; 4) orientation of this ellipse (its minor axis is located in the meridian plane, at $14.5 \mathrm{~W}$ degrees).

The influence of the dissipation on the damping of the Chandler polar motion was studied. The analytical solution of the problem was obtained for the simplest treatment of the delay of the tides caused by the Earth's rotation (Getino \& Ferrándiz 1991; Kubo, 1991). This model explains the characteristic behaviour of the amplitude of the Chandler motion in the periods 1905-1920, 1943-1960 (Vondrák, \& Cyril, 1966). The excitation of the Chandler motion can be explained by the upper and lower envelope displacements (Barkin, 1999) with Moon-Sun forced attraction with a period of 412 days, close to the Chandler period.

\section{Equations of Motion}

Consider the Earth as the weakly deformable body, assuming that its particles in the process of the body motion are weakly displaced from their initial positions. The body has an inner rigid envelope, with which we connect the Cartesian reference system $C \xi \eta \zeta$, and an external deformable envelope. The origin of these reference systems is the center of mass of the body. We will suggest that 
axes of this reference system coincide with corresponding axes of inertia of the body in its undeformed state.

Let $C x y z$ be the main reference system with the same origin and with axes having their permanent orientation in space. We define the orientation and the motion of the axes $C \xi \eta \zeta$ with respect to the reference system $C x y z$ by Andoyer variables $G, \theta, \rho, l, g, h$ (Getino \& Ferrándiz 1990; Barkin, 1996). Let $\bar{\omega}$ be the angular velocity vector of rotation of the body reference system $C \xi \eta \zeta$ in the main reference system $C x y z$. Its projections on the axes $C \xi, C \eta$, and $C \zeta$ are $p, q, r$.

Let the body be isolated and no forces act on it. In this case the external moment equals zero and the equations of its rotational motion in Andoyer variables $G, \theta, \rho, l, g, h$ can be written in the following way (Barkin, in press):

$$
\begin{aligned}
\frac{d G}{d t}= & 0, \frac{d \rho}{d t}=0, \frac{d h}{d t}=0, \\
\frac{d \theta}{d t}= & G \sin \theta\left[\frac{1}{2}(a-b) \sin 2 l-f \cos 2 l\right]+G \cos \theta(d \sin l-e \cos l)+ \\
& \beta \sin l-\alpha \cos l, \\
\frac{d l}{d t}= & G \cos \theta\left(c-a \sin ^{2} l-b \cos ^{2} l+f \sin 2 l\right)+ \\
& G \csc \theta \cos 2 \theta(e \sin l+d \cos l)-\gamma+\cot \theta(\alpha \sin l+\beta \cos l), \\
\frac{d g}{d t}= & G\left(a \sin ^{2} l+b \cos ^{2} l-f \sin 2 l\right)+ \\
& G \cot \theta(e \sin l+d \cos l)-\csc \theta(\alpha \sin l+\beta \cos l),
\end{aligned}
$$

where

$$
\begin{aligned}
a & =\frac{B C-D^{2}}{\Delta}, b=\frac{A C-E^{2}}{\Delta}, c=\frac{A B-F^{2}}{\Delta}, \\
f & =-\frac{E D+F C}{\Delta}, e=-\frac{F D+B E}{\Delta}, d=-\frac{F E+A D}{\Delta}, \\
\Delta & =A B C-A D^{2}-B E^{2}-C F^{2}-2 F E D .
\end{aligned}
$$

In (2) $A, B, C$ and $F, E, D$ are axial and centrifugal moments of inertia, which are definite functions of time and Andoyer variables; $\alpha, \beta, \gamma$ are the components of some angular velocity:

$$
\begin{aligned}
\alpha & =a P-f Q-e R, \\
\beta & =-f P+b Q-d R, \\
\gamma & =-e P-d Q+c R,
\end{aligned}
$$

and $P, Q, R$ are the components of the relative angular momentum of the particle motion of the body in its frame.

In the case considered, the first three equations of (1) are separated from the general set and give three first integrals: $G=G_{0}, \rho=\rho_{0}, h=h_{0}$ implying that the angular momentum vector of the rotational motion of deformable celestial bodies is a constant. $G_{0}, \rho_{0}$ and $h_{0}$ are initial values of the corresponding Andoyer variables. 
We will use some simplifications and reductions of the exact equations (1)(3) on the basis of some additional assumptions. To concentrate our attention on the effects pointed out in this paper we will suppose that the angular momentum of the relative motion of the body particles is equal to zero $(P=Q=R=0)$. Assuming now that the body is weakly deformable, we can use the following representations for the moments of inertia:

$$
\begin{array}{ccc}
A=A_{0}+A_{1}, & B=3 D B_{0}+B_{1}, & C=3 D C_{0}+C_{1}, \\
F=F_{0}+F_{1}, & E=3 D E_{0}+E_{1}, & D=3 D D_{0}+D_{1},
\end{array}
$$

where $A_{0}, B_{0}, C_{0}$ are the principal moments of inertia for the undeformed state of the body (in our reference system $C \xi \eta \zeta$ we have $F_{0}=0, E_{0}=0, D_{0}=0$ ), and $A_{1}, B_{1}, C_{1} ; F_{1}, E_{1}, D_{1}$ are small perturbations of these moments due to its rotational deformation.

Using representations of the main characteristics of the body (4) we can present the equations (1) in following approximate form:

$$
\begin{aligned}
\frac{d \theta}{d t}= & G \sin \theta\left\{\frac{1}{2}\left[\frac{1}{A_{0}}-\frac{1}{B_{0}}+\frac{B_{1}}{B_{0}^{2}}-\frac{A_{1}}{A_{0}^{2}}\right] \sin 2 l+\frac{F_{1}}{A_{0} B_{0}} \cos 2 l\right\}+ \\
& +\frac{G}{C_{0}} \cos \theta\left(\frac{E_{1}}{A_{0}} \cos l-\frac{D_{1}}{B_{0}} \sin l\right), \\
\frac{d l}{d t}= & G \cos \theta\left\{\frac{1}{C_{0}}-\frac{C_{1}}{C_{0}}-\left(\frac{1}{A_{0}}-\frac{A_{1}}{A_{0}^{2}}\right) \sin ^{2} l-\left(\frac{1}{B_{0}}-\frac{B_{1}}{B_{0}^{2}}\right) \cos ^{2} l-\right. \\
& \left.-\frac{F_{1}}{A_{0} B_{0}} \sin 2 l\right\}-\frac{G}{C_{0}} \csc \theta \cos 2 \theta\left(\frac{E_{1}}{A_{0}} \sin l+\frac{D_{1}}{B_{0}} \cos l\right), \\
\frac{d g}{d t}= & G\left[\left(\frac{1}{A_{0}}-\frac{A_{1}}{A_{0}^{2}}-\right) \sin ^{2} l+\left(\frac{1}{B_{0}}-\frac{B_{1}}{B_{0}^{2}}\right) \cos ^{2} l-\frac{F_{1}}{A_{0} B_{0}} \sin 2 l\right]+ \\
& +\frac{G}{C_{0}} \cot \theta\left(\frac{E_{1}}{A_{0}} \sin l+\frac{D_{1}}{B_{0}} \cos l\right) .
\end{aligned}
$$

If we obtain some solution of the equations (5) in Andoyer variables we can study also the corresponding effects for components of the angular velocity of the body frame rotation. The projections $p, q, r$ of this vector on the axes of the body frame $C \xi \eta \zeta$ are given by the following formulae:

$$
\begin{aligned}
& p=G[\sin \theta(a \sin l-f \cos l)-e \cos \theta]-\alpha, \\
& q=G[\sin \theta(-f \sin l+b \cos l)-d \cos \theta]-\beta, \\
& r=G[\sin \theta(-e \sin l-d \cos l)+c \cos \theta]-\gamma,
\end{aligned}
$$

where coefficients are defined as definite functions of time by the formulae (2), (3).

\section{Chandler's Unperturbed Motion and its Properties}

For variations of the components of the Earth's inertia tensor due to its rotation we will use known classical expressions. Neglecting small terms of order $\mu \sin ^{2} \theta$ 
in a simplified variant of the problem, we have (Getino \& Ferrándiz 1991):

$$
\begin{aligned}
& A_{1}=-\mu C, B_{1}=-\mu C, C_{1}=2 \mu C, \\
& F_{1}=0, E_{1}=-3 \mu C \sin \theta \cos \theta \sin l, \\
& D_{1}=-3 \mu C \sin \theta \cos \theta \cos l,
\end{aligned}
$$

where $\mu$ is some small elastic parameter of the Earth $\left(\mu=0.3364 \cdot 10^{-3}\right.$, Getino \& Ferrándiz (1991)).

Substituting formulae (7) into equations (5), we retain the main terms in the right-hand sides of these equations. Let us conserve the terms of the first order with respect to $\mu$, but neglect the terms of the third order with respect to small parameters: $\mu,\left(A_{0}-B_{0}\right) / A_{0}, \theta$ (in reality, we add these small terms to the perturbing terms in the right-hand sides of the equations).

As a result of simple transformations, the equations of the rotational motion of the isolated celestial body can be presented in the following compact form:

$$
\begin{aligned}
& \frac{d \theta}{d t}=G \sin \theta \sin l \cos l\left(\frac{1}{A_{0}}-\frac{1}{B_{0}}\right)(1-2 \mu) \\
& \frac{d l}{d t}=G \cos \theta\left[\frac{1}{C_{0}}-\left(\frac{\sin ^{2} l}{A_{0}}+\frac{\cos ^{2} l}{B_{0}}\right)\right](1-2 \mu) \\
& \frac{d g}{d t}=G\left(\frac{\sin ^{2} l}{A_{0}}+\frac{\cos ^{2} l}{B_{0}}\right)(1-2 \mu) .
\end{aligned}
$$

Here $A_{0}, B_{0}$ and $C_{0}$ are principal moments of inertia of the body in the undeformed state.

Equations (8) fully coincide with the equations of the Euler-Poinsot problem described in the Andoyer variables for an absolutely rigid body with principal moment of inertia:

$$
\tilde{A}=A_{0}(1+2 \mu), \tilde{B}=B_{0}(1+\dot{2} \mu), \tilde{C}=C_{0}(1+2 \mu) .
$$

In the deformed state (in the observed rotational motion of the Earth) the average axial moments of inertia are

$$
\bar{A}=A_{0}(1-\mu), \bar{B}=B_{0}(1-\mu), \bar{C}=C_{0}(1+2 \mu) .
$$

This means that the moments of inertia (9) are different from the real values (10) and are connected to them by simple relationships:

$$
\tilde{A}=\overline{A_{0}}(1+3 \mu), \tilde{B}=\bar{B}_{0}(1+3 \mu), \tilde{C}=\bar{C}_{0} .
$$

The rotational motion of an elastic body deformed by its own rotation is executed according on the Euler-Poinsot laws for an equivalent rigid body with different principal central moments of inertia: $\tilde{A}=\bar{A}(1+3 \mu), \tilde{B}=$ $\bar{B}(1+3 \mu), \tilde{C}=\bar{C}$, where $\mu$ is the coefficient of elasticity, and $\bar{A}, \bar{B}$ and $\bar{C}$ are average values of the principal moments of inertia of the rotating body.

In other words the elastic body rotates as an absolutely rigid body, but with equatorial moments of inertia increased by $3 \mu C_{0}$. The polar moment of inertia 
of the model body is equal to the mean moment of inertia of the deformable body.

Solution of the equations (8) in the elliptic functions and their detailed studies led us to establish the following important properties of the unperturbed Chandler motion (Barkin et al. 1995; Barkin, 1996).

1. The projection of the trajectory of the end of the angular velocity vector $\omega$ on the equatorial plane of the body $C \xi \eta$ is an ellipse with eccentricity $e=0.0958$ and with the minor semi-axis directed parallel to the principal central axes of inertia of the Earth, corresponding to the maximum from equatorial moments of inertia (this axis is located 14.5 degrees west of the Greenwich meridian).

2. The mean Chandler frequency of the motion along the ellipse $\Omega=-2.3086$. $10^{-3} \omega_{0}$ ( $\omega_{0}$ is the mean value of the angular velocity of the Earth) defines the straight polar motion (in the counter clockwise direction, if viewed from the end of the Earth's polar axis $\left(C_{\zeta}\right)$ ) with a period of 433.11 days.

3 . The polar motion along the ellipse is executed with a variable velocity. The maximum velocity is achieved at the moment of crossing of the smallest of the equatorial axes of the Earth's ellipsoid of inertia (the corresponding Chandler's period is 433.08 days), and the minimum velocity is achieved at the moment of crossing of the major equatorial axes (the corresponding value of Chandler's period is 437.11 days). This means that the variation of the conditional Chandler period of the polar motion is 4.03 days.

\section{Damping of the Chandler polar motion.}

Now we study effects of damping of the Chandler motion on the basis of the simplest model. Differential equations of this model follow from general equations and have form (here we use notations $A=A_{0}=B_{0}, C=C_{0}$, see section 1.):

$$
\begin{aligned}
i & =L\left(\frac{1}{C}-\frac{1}{A}+\frac{3 \mu}{A} \cos \delta\right), \\
\dot{\theta} & =-\frac{3 \mu}{A} G \sin \theta \sin \delta, \\
\dot{g} & =\frac{G}{A}(1-3 \mu \cos \theta \cos \delta),
\end{aligned}
$$

where $\delta$ is the angle of delay (Getino \& Ferrándiz 1991; Kubo, 1991).

Solution of equations (12) is

$$
\begin{aligned}
\cos \theta & =\sqrt{1-\sin ^{2} \theta_{0} e^{-2 \alpha l}}, \\
\tan \theta & =\frac{\sin \theta_{0} e^{-\alpha l}}{\sqrt{1-\sin ^{2} \theta_{0} e-2 \alpha l}}, \quad l=\frac{1}{2 \alpha} \ln \left[\frac{1-\tan ^{2} \Omega\left(t-t_{0}\right)}{\sin ^{2} \theta_{0}}\right], \\
g & =\frac{G}{A}\left(t-t_{0}\right)+\frac{3 G \mu}{A \Omega} \cos \delta \ln \left|\cos \Omega\left(t-t_{0}\right)\right|+g_{0}
\end{aligned}
$$


where $\Omega=G\left(\frac{1}{C}-\frac{1}{A}+\frac{3 \mu}{A} \cos \delta\right)$ is the Chandler frequency.

For values of the angle $l=2 \pi k$ with $k$ an integer, we obtain the relation of the two consequent amplitudes $\sin \theta_{k} / \sin \theta_{k-1}=e^{-2 \pi \alpha}$.

From typical behaviour of the trajectory of the Chandler polar motion in the period 1985-1995 (IERS report 1997) it is easy to obtain the following evaluation of the parameter $\alpha \simeq 0.036$. The value $\mu$ we can also evaluate from $\alpha$ :

$$
\mu=\frac{\alpha\left(\frac{A-C}{C}\right)}{\sin \delta-\alpha \cos \delta} \approx \frac{C-A}{C},
$$

so the angle of delay $\delta \approx 1^{\circ}$ in accordance with Kubo (1991).

In the Earth's case the angle $\theta \approx 10^{-6}$ is very small. Neglecting terms of second order $\approx \mu \theta^{2}$ we can simplify the equations (12):

$$
\begin{aligned}
\frac{d \theta}{d t} & =-\theta D \sin \delta \\
\frac{d l}{d t} & =G\left(\frac{1}{C}-\frac{1}{2}\left(\frac{1}{A}+\frac{1}{B}\right)\right)+\frac{3 \mu G}{A} \cos \delta=\Omega \\
\frac{d g}{d t} & =\frac{G}{2}\left(\frac{1}{A}+\frac{1}{B}\right)-\frac{3 \mu G}{A} \cos \delta=\omega
\end{aligned}
$$

where $D=3 \omega_{0} \mu, \omega_{0}=\frac{G}{A} ; \Omega$ and $\omega$ are basic frequencies.

The solution of the equations (14) are defined by very simple formulae:

$$
\theta=-\theta_{0} e^{-D \sin \delta\left(t-t_{0}\right)}, \quad l=\Omega\left(t-t_{0}\right)+l_{0}, \quad g=\omega\left(t-t_{0}\right)+g_{0},
$$

where $\theta_{0}, l_{0}$ and $g_{0}$ are initial values of the Andoyer variables $\theta, l$ and $g$.

The characteristic time to decrease the exponent of the angle $\theta$ by a factor of two is

$$
T_{D}=\frac{\ln 2}{D \sin \delta}=\frac{2 \pi}{\omega_{0}} 6.2641 \cdot 10^{3}=17.15 \text { years } .
$$

In accordance with Vondrák \& Cyril (1997) characteristic damping of the Chandler motion of the Earth's pole took place in the periods of time 1935-1957 and 1975-1990. It was executed approximately with similar amplitude and with good agreement with determined period $T_{D}$.

\section{About Possible Mechanism of Excitation of the Pole Motion}

To explain excitation of the Earth's polar motion a new mechanism of the Earth layer displacements (Barkin, 1999) is suggested. According to the new approach, the Earth's layers are considered as nonspherical inhomogeneous celestial bodies, interacting with each other, with the Moon and Sun and executing slow relative motions. Of primary importance here are slow and small displacements of the centers of masses of the lower and upper mantles (boundary at $670 \mathrm{~km}$ ) and their slow relative rotations. These displacements display themselves at various time scales (from a few months to millions of years), and their manifestations are readily detected in the regularities of the distribution of geological structures as well as in many geodynamical processes (Barkin, 1999). 
The layer motions and the accompanying tectonic mass redistribution cause variations of the components of the Earth's inertia tensor and geopotential, leading to variations of polar motion. In accordance with our preliminary studies, the excitation of polar motion is caused by displacements of the lower Earth layer with a period of 412 days, close to the Chandler period due to corresponding lunisolar perturbations. It is a source for excitation of the Chandler polar motion and in particular it defines its long-periodic behaviour. Preliminary analytical description of the specific phenomena on the basis of a non-resonant model was given. Elementary explanation of the observed forced Chandler motion are given by Barkin elsewhere in the proceedings.

Acknowledgments. JMF's work has been partially supported by Spanish Projects ESP97-1816-C04-02 (CICYT) and PB95-696 (DGES). Yu.B's stay at University of Alicante has been partially funded by grant INV99-01-06 Generalitat Valenciana.

\section{References}

Barkin, Yu.V., Ferrándiz, J.M \& Getino, J. 1995, Abstracts Book. IAU Symposium N172. Dynamics, Ephemerides and Astrometry in the Solar System (Paris, France, July 3-8), p. 44.

Barkin, Yu.V. 1996, Proceedings of the International Conference "Earth Rotation. Reference Systems in Geodynamics and Solar System" (Warsaw, Poland, September 18-20, 1995). Journées 1995. SRC, PAS, Warsaw, Poland, 1996, pp. 83-86.

Barkin, Yu.V. 1998, Astronomical and Astrophysical Transactions, 17, 179-219.

Barkin, Yu.V. 1999, Proceedings of Conference "Interaction in the LithosphereHydrosphere-Atmosphere System", Moscow, November 28-29, pp. 46-60. In Russian

Barkin, Yu.V. (in press), Astronomical and Astrophysical Transactions.

IERS 1995, Annual Report, Int. Earth Rotation Service, Paris.

Ferrándiz, J.M. \& Getino, J. 1993, Celestial Mechanics and Dynamical Astronomy, 57, 279-292.

Getino, J., \& Ferrándiz, J.M. 1990, Celestial Mechanics, 49, 303-326.

Getino, J. \& Ferrándiz, J.M. 1991, Celestial Mechanics, 52, 381-396.

Kubo, Y. 1991, Celestial Mechanics, 50, 165-187.

Vondrák, J. \& Cyril, R. 1996, Procedure of the International Conference "Earth Rotation reference Systems in Geodynamics and Solar System". Warsaw, Poland, September 18-20, 1995. Journées 1995, SRC, PAS. Warsaw, Poland, pp. 95-102. 